EHU-96-4

July 14, 2018

\title{
Is Zee Model The Model of Neutrino Masses?
}

\author{
Alexei Yu. Smirnov ${ }^{a}$ 丹, Morimitsu Tanimoto, ${ }^{b, 2}$ \\ a International Center for Theoretical Physics \\ I-34100 Trieste, Italy \\ ${ }^{b}$ Science Education Laboratory, Ehime University, \\ Matsuyama 790, JAPAN
}

\begin{abstract}
Zee model leads naturally to two heavy, strongly degenerate and almost maximally mixed neutrinos and to one light neutrino with small mixing. This pattern coincides with the one needed for a solution of the atmospheric neutrino problem by $\nu_{\mu} \rightarrow \nu_{\tau}$ oscillations and for existence of the two component hot dark matter in the Universe. Furthermore, the oscillations $\bar{\nu}_{\mu} \rightarrow \bar{\nu}_{e}$ can be in the range of sensitivity of KARMEN, LSND experiments. Phenomenology of this scenario is considered and possibility to check it in the forthcoming experiments is discussed. Scenario implies large values and inverse flavour hierarchy of the couplings of the Zee boson with fermions: $f_{e \tau} \ll f_{\mu \tau} \leq f_{e \mu} \sim 0.1$. Main signatures of scenario are: strongly suppressed signal of $\nu_{\mu} \rightarrow \nu_{\tau}$ oscillation in CHORUS and NOMAD experiments, so that positive result from these experiments will rule out the scenario; possibility of observation of $\nu_{e} \rightarrow \nu_{\tau}$ oscillations by CHORUS and NOMAD; corrections to the muon decay, neutrinoelectron scattering at the level of the experimental errors; branching ratio $B(\mu \rightarrow e \gamma)$ bigger than $10^{-13}$. The solar neutrino problem can be solved by introduction of additional very light singlet fermion without appreciable changes of the active neutrino pattern.
\end{abstract}

\footnotetext{
${ }^{1}$ E-mail: smirnov@ictp.trieste.it

${ }^{2}$ E-mail: tanimoto@edserv.ed.ehime-u.ac.jp
} 


\section{Introduction}

Zee model [四] is the simplest model which explains a smallness of neutrino masses by physics at the electroweak scale. It can be considered as an alternative of the see-saw mechanism [2].

Zee model suggests the existence of charged scalar field $h$, singlet of the $S U(2)$, and two doublets of the Higgs bosons $\Phi_{1}, \Phi_{2}$. The singlet $h$ couples to lepton doublets $\Psi_{l L}=\left(\nu_{l}, l^{-}\right),(l=e, \mu, \tau)$ as well as to Higgs doublets; leptons are assumed to couple to doublet $\Phi_{1}$ only. The appropriate terms in the Lagrangian are

$$
\mathcal{L}_{Z e e}=f_{\ell \ell^{\prime}} \Psi_{\ell L}^{T} i \tau_{2} \Psi_{\ell^{\prime} L} h+c_{12} \Phi_{1}^{T} i \tau_{2} \Phi_{2} h^{\dagger}+\frac{m_{l}}{\left\langle\Phi_{1}\right\rangle} \bar{\Psi}_{l} \Phi_{1} l_{R}+\text { h.c. }
$$

where $c_{12}=-c_{21}$ are real mass parameters, the couplings $f_{\ell \ell^{\prime}}$ are antisymmetric in $\ell$ and $\ell^{\prime}$. The interactions (迎) generate neutrino mass terms in one loop.

Zee model gives very distinctive pattern of neutrino masses and mixing. For not too strong hierarchy of the couplings $f_{\ell \ell^{\prime}}$ the two heavy neutrinos, $\nu_{2}, \nu_{3}$, are strongly degenerate and mix almost maximally in $\nu_{\mu}, \nu_{\tau}$. The first neutrino $\nu_{1}$ practically coincides with $\nu_{e}$ and has much smaller mass:

$$
m_{1} \ll m_{2} \approx m_{3}
$$

It was marked [3] that this pattern coincides with the one needed to solve simultaneously the atmospheric neutrino problem [4] and the problem of the hot dark matter in the Universe [5]. Indeed, the deficit of the atmospheric muon neutrinos can be explained by the oscillations $\nu_{\mu}-\nu_{\tau}$ with practically maximal mixing. Two heavy neutrinos with masses $m_{2} \approx m_{3} \approx(1-5) \mathrm{eV}$ compose two component hot dark matter (which may give even better fit of the cosmological data than one component) [5]. Furthermore, the oscillations $\nu_{\mu} \rightarrow \nu_{e}$ and $\bar{\nu}_{\mu} \rightarrow \bar{\nu}_{e}$ can be at the level of sensitivity

of existing experiments: BNL [6], KARMEN [7] (see [3]). Later it was marked [8] [9] 
that the model can immediately accommodate positive LSND result [10].

In this paper we will consider phenomenology of the outlined scenario, and in particular, the possibility to check it by forthcoming experiments. In sect. 2 we describe the scenario in details. Sect. 3 is devoted to oscillations. In sect. 4 we find the bounds on the Zee coupling constants. In sect. 5 implications of data on the muon decay, neutrino electron scattering, $e-\mu-\tau$ universality to the scenario are considered. Predictions for $\mu \rightarrow e \gamma$ and $\nu_{3(2)} \rightarrow \nu_{1} \gamma$ are given. In sect. 6, we describe a modification of the Zee model which is able to solve the solar neutrino problem. Sect. 7 contains our conclusions.

\section{Scenario}

The neutrino mass matrix of the Zee model in flavor basis, $\nu=\left(\nu_{e}, \nu_{\mu}, \nu_{\tau}\right)$, can be written as

$$
M=m_{0}\left(\begin{array}{ccc}
0 & \epsilon & \sin \theta \\
\epsilon & 0 & \cos \theta \\
\sin \theta & \cos \theta & 0
\end{array}\right)
$$

where $m_{0}$ is the basic mass scale. Mixing angle $\theta$ and parameter $\epsilon$ can be naturally much smaller than 1. (We will discuss the relation of these parameters with the parameters of the Lagrangian (1) in sect. 4.)

In the case $\cos \theta \gg \sin \theta, \epsilon$ the eigenvalues of matrix (3) are

$$
m_{1}=-m_{0} \epsilon \sin 2 \theta, \quad m_{2,3}=m_{0}\left( \pm 1-\frac{1}{2} \epsilon \sin 2 \theta\right)
$$

and the mixing matrix $S$ which diagonalizes (3) is

$$
S \simeq \frac{1}{\sqrt{2}}\left(\begin{array}{ccc}
\sqrt{2} \cos \theta & \sin \theta+\epsilon \cos \theta & \sin \theta-\epsilon \cos \theta \\
-\sqrt{2} \sin \theta & \cos \theta & \cos \theta \\
-\sqrt{2} \epsilon & 1 & -1
\end{array}\right)
$$


$\left(\nu_{f}=S \nu\right.$, where $\nu \equiv\left(\nu_{1}, \nu_{2}, \nu_{3}\right)$ are the mass eigenstates). According to (田) the states $\nu_{2}$ and $\nu_{3}$ are approximately degenerate, and their masses $\left(\sim m_{0}\right)$ are much larger than the mass of $\nu_{1}$. The mass squared difference is

$$
\Delta m_{32}^{2}=2 \epsilon \sin 2 \theta m_{0}^{2} \ll m_{0}^{2},
$$

where $\Delta m_{i j}^{2} \equiv m_{i}^{2}-m_{j}^{2}$. For $\nu_{1}$ component model gives $\Delta m_{21}^{2} \simeq \Delta m_{31}^{2} \simeq m_{0}^{2}$ and the ratio of mass differences equals

$$
\frac{\Delta m_{32}^{2}}{\Delta m_{21}^{2}}=2 \epsilon \sin 2 \theta
$$

Thus, the Zee mass matrix gives two different scales for the mass squared differences and the maximal mixing between two heaviest neutrinos.

As it was outlined in the introduction we will consider the following scenario:

- $\nu_{2}$ and $\nu_{3}$ form the two component hot dark matter so that

$$
m_{0}=m_{H D M}=(1-5) \mathrm{eV} .
$$

- The $\nu_{\mu}-\nu_{\tau}$ oscillations with practically maximal depth explain the atmospheric neutrino deficit and therefore

$$
\Delta m_{32}^{2}=\Delta m_{a t m}^{2} \sim(0.3-3) \cdot 10^{-2} \mathrm{eV}^{2} .
$$

- The oscillations $\nu_{\mu}-\nu_{e}$ and $\bar{\nu}_{\mu}-\bar{\nu}_{e}$ with $\Delta m_{21}^{2} \approx m_{H D M}^{2}$ can be in the region of sensitivity of the KARMEN and LSND experiments. For $m_{0}$ in the cosmologically interesting domain (8) this means that

$$
\sin ^{2} 2 \theta \leq \sin ^{2} 2 \theta_{e \mu} \sim(1-3) \cdot 10^{-3},
$$

where $\theta_{e \mu}$ is the experimental bound (or preferable value in the case of positive result) for the $\nu_{e}-\nu_{\mu}$ mixing angle. 
Substituting $m_{0}$ and $\sin ^{2} 2 \theta$ from (8) and (10) in (6) we get

$$
\epsilon \geq \frac{\Delta m_{a t m}^{2}}{2 m_{H D M}^{2} \sin 2 \theta_{e \mu}} .
$$

According to (9) and (11) $\epsilon=10^{-3}-0.5$, with typical value $3 \cdot 10^{-2}$. Thus all oscillation parameters of the model (mass squared differences and mixing angles) can be fixed by the experimental data immediately.

\section{Neutrino Oscillations}

In terms of the elements of the mixing matrix (5), $S_{\alpha i}$, the oscillation probability can be written as

$$
P\left(\nu_{\alpha} \rightarrow \nu_{\beta}\right)=\delta_{\alpha \beta}-4 \sum_{i>j} S_{\alpha i} S_{\beta i} S_{\alpha j} S_{\beta j} \sin ^{2}\left(\frac{\Delta m_{i j}^{2} L}{4 E_{\nu}}\right),
$$

where $E_{\nu}$ is the neutrino energy and $L$ is the distance. We neglect the $C P$ violation, so that the elements $S_{\alpha i}$ are real. Let us consider the probabilities (12) for short and long distances separately.

1). In the short distance limit the phase difference due to $\Delta m_{32}^{2}$ is small: $\frac{\Delta m_{32}^{2} L}{4 E_{\nu}} \ll$ 1. Taking into account that $\Delta m_{31}^{2} \simeq \Delta m_{21}^{2}$ and using matrix (5) we find

$$
P\left(\nu_{\mu} \rightarrow \nu_{e}\right) \approx P\left(\bar{\nu}_{\mu} \rightarrow \bar{\nu}_{e}\right)=\sin ^{2} 2 \theta \sin ^{2}\left(\frac{\Delta m_{31}^{2} L}{4 E_{\nu}}\right) .
$$

This result is applied to E776 [6], KARMEN [7] and LSND [10].

For $\nu_{\mu}-\nu_{\tau}$ we get

$$
P\left(\nu_{\mu} \rightarrow \nu_{\tau}\right)=4(\epsilon \sin \theta)^{2} \sin ^{2}\left(\frac{\Delta m_{31}^{2} L}{4 E_{\nu}}\right)+\cos ^{2} \theta \sin ^{2}\left(\frac{\Delta m_{32}^{2} L}{4 E_{\nu}}\right) .
$$

The $\nu_{\mu}-\nu_{\tau}$ oscillations with large mass splitting are doubly suppressed because of $\sin \theta \ll 1$ and $\epsilon \ll 1$. This smallness is related to the fact that according to (5) the admixture of $\nu_{1}$ in $\nu_{\tau}$ is suppressed by $\epsilon$. The mode of oscillations with the smallest 
mass splitting (second term in (14)) may give a comparable contribution. For values of $\sin ^{2} 2 \theta$ and $\epsilon$ from (10) and (11) correspondingly we obtain

$$
P\left(\nu_{\mu} \rightarrow \nu_{\tau}\right) \sim 10^{-7}-10^{-5}
$$

If both $\epsilon$ and $\Delta m^{2}$ are near the upper bounds the probability can be as big as $10^{-4}$. These values are still below the sensitivity of CHORUS and NOMAD [1], but they may be in the regions of sensitivity of planning experiments E803 at Fermilab and E889 at BNL [12].

For $\nu_{e}-\nu_{\tau}$ channel we get

$$
P\left(\nu_{e} \rightarrow \nu_{\tau}\right)=4(\epsilon \cos \theta)^{2} \sin ^{2}\left(\frac{\Delta m_{31}^{2} L}{4 E_{\nu}}\right)
$$

If $\epsilon \geq 10^{-1}$ and $m_{0}>4 \mathrm{eV}$, then $P\left(\nu_{e} \rightarrow \nu_{\tau}\right) \simeq 10^{-2}-10^{-1}$ and the $\nu_{e} \rightarrow \nu_{\tau}$ oscillations can be detected by CHORUS and NOMAD.

Thus the observation of signals of the $\nu_{e} \rightarrow \nu_{\tau}$ oscillation and absence of signal from $\nu_{\mu} \rightarrow \nu_{\tau}$ mode in CHORUS and NOMAD are the signatures of the Zee model. The scenario under consideration will be ruled out if CHORUS and NOMAD find signals of the $\nu_{\mu} \rightarrow \nu_{\tau}$ oscillations.

According to (6) for fixed $\Delta m_{32}^{2}$ and $m_{0}$, the parameter $\epsilon$ is inversely proportional to $\sin \theta$. Therefore $P\left(\nu_{e} \rightarrow \nu_{\tau}\right)$ increases when $P\left(\nu_{\mu} \rightarrow \nu_{e}\right)$ decreases, as is shown in Fig.1.

\section{Fig. 1}

In particular, if $P\left(\bar{\nu}_{\mu} \rightarrow \bar{\nu}_{e}\right) \sim 3 \cdot 10^{-3}$ (the level of the LSND result) and $m_{0}^{2}>6$ $\mathrm{eV}^{2}$, then $P\left(\nu_{e} \rightarrow \nu_{\tau}\right)<3 \cdot 10^{-5}$ which is beyond the sensitivity of the CHORUS and NOMAD. On the contrary, for $P\left(\nu_{e} \rightarrow \nu_{\tau}\right)>2 \cdot 10^{-2}$ which can be observed by these 
experiments one has $P\left(\nu_{\mu} \rightarrow \nu_{e}\right)<10^{-5}$. Thus a comparison of results from searches for $P\left(\nu_{e} \rightarrow \nu_{\tau}\right)$ and $P\left(\nu_{e} \rightarrow \nu_{\mu}\right)$ oscillations can give crucial check of the model.

For the parameters under consideration there are strong resonance transitions $\nu_{e} \rightarrow \nu_{\mu}, \nu_{\tau}$ and $\nu_{\mu} \rightarrow \nu_{e}$ in the inner parts of the collapsing stars. As the consequences one predicts: (i) disappearance of the neutronization peak, (ii) hard $\nu_{e}$ spectrum at the cooling stage, (iii) additional energy release in the inner parts of star which will stimulate shock wave revival desired for the star explosion. (iv) In the same time the $\nu_{\mu} \rightarrow \nu_{e}$ conversion leads to suppression of the r-processes responsible for nucleosynthesis of heavy elements unless $m_{0} \leq 2 \mathrm{eV}$ [13.

2). In the long distance limit experiments are sensitive to oscillations stipulated by small mass difference $\Delta m_{32}^{2}$ and the oscillations due to large mass difference are averaged out. We get the results

$$
\begin{aligned}
P\left(\nu_{\mu} \rightarrow \nu_{e}\right) & =\frac{1}{2}(\sin 2 \theta)^{2}-\cos ^{2} \theta\left(\sin ^{2} \theta-\epsilon^{2} \cos ^{2} \theta\right) \sin ^{2}\left(\frac{\Delta m_{32}^{2} L}{4 E_{\nu}}\right) \\
P\left(\nu_{\mu} \rightarrow \nu_{\tau}\right) & =2(\epsilon \sin \theta)^{2}+\cos ^{2} \theta \sin ^{2}\left(\frac{\Delta m_{32}^{2} L}{4 E_{\nu}}\right)
\end{aligned}
$$

which are applied to the atmospheric neutrinos. Notice that $P\left(\nu_{\mu} \rightarrow \nu_{e}\right)$ is suppressed due to both $\sin \theta \ll 1$ and $\epsilon \ll 1$, and the dominant effect comes from $\nu_{\mu} \rightarrow \nu_{\tau}$ oscillations as we suggested in the introduction.

\section{Parameters of Zee Model}

In terms of parameters of the Lagrangian (11) the elements of the mass matrix (3) equal

$$
\tan \theta \equiv \frac{f_{e \tau}}{f_{\mu \tau}}, \quad \epsilon \equiv \frac{f_{e \mu}}{\sqrt{f_{e \tau}^{2}+f_{\mu \tau}^{2}}}\left(\frac{m_{\mu}}{m_{\tau}}\right)^{2}
$$


and [1] [14]

$$
m_{0} \approx m_{\tau}^{2} \sqrt{f_{e \tau}^{2}+f_{\mu \tau}^{2}} \frac{g \sin 2 \phi \cot \beta}{64 \sqrt{2} M_{W} \pi^{2}} \ln \frac{M_{2}^{2}}{M_{1}^{2}}
$$

Here $m_{\tau}$ is the tau lepton mass, $g$ is the weak coupling, $m_{W}$ is the $W$-boson mass, $\tan \beta \equiv<\Phi_{1}>_{0} /<\Phi_{2}>_{0}$ is the ratio of the VEV of two Higgs doublets. The angle $\beta$ determines physical charged Higgs boson: $\Phi^{+}=\Phi_{1}^{+} \cos \beta-\Phi_{2}^{+} \sin \beta$, where $\Phi_{1}^{+}, \Phi_{2}^{+}$ are two charged Higgs fields from the doublets. The angle $\phi$ is the mixing angle of the Zee singlet and the physical charged component of the Higgs doublet $\Phi^{+}$:

$$
\begin{gathered}
h=\cos \phi H_{1}+\sin \phi H_{2}, \\
\Phi^{+}=-\sin \phi H_{1}+\cos \phi H_{2},
\end{gathered}
$$

where $H_{1}$ and $H_{2}$ are the eigenstates of the mass matrix with masses $M_{1}$ and $M_{2}$, and the mixing angle is determined by

$$
\tan 2 \phi=\frac{4 \sqrt{2} g^{-1} c_{12} M_{W}}{\sqrt{\left(M_{1}^{2}-M_{2}^{2}\right)^{2}-\left(4 \sqrt{2} g^{-1} c_{12} M_{W}\right)^{2}}} .
$$

As we have seen in sect. 2, the parameters of the mass matrix (3) $m_{0}, \epsilon, \theta$ can be fixed by the data. This in turn allows one to determine the ratios of the constants $f_{i j}$ using (19)

$$
\frac{f_{e \tau}}{f_{\mu \tau}}=\tan \theta_{e \mu} \ll 1,
$$

and

$$
\frac{f_{e \mu}}{f_{\mu \tau}} \approx \frac{\Delta m_{a t m}^{2}}{2 m_{H D M}^{2}} \cdot\left(\frac{m_{\tau}}{m_{\mu}}\right)^{2} \cdot \frac{1}{\sin 2 \theta_{e \mu}} .
$$

For $\sin ^{2} 2 \theta_{e \mu}=2 \times 10^{-3}, \Delta m_{H D M}^{2}=6 \mathrm{eV}^{2}$ and $\Delta m_{\text {atm }}^{2}=10^{-2} \mathrm{eV}^{2}$ Eq. (24) gives $f_{e \mu} / f_{\mu \tau}=5.3$ which means an inverse hierarchy of the Zee boson couplings with $f_{e \mu}$ being the largest one [9]. For fixed value $P\left(\nu_{\mu} \rightarrow \nu_{e}\right)$ the mixing angle $\theta$ is the function of $\Delta m_{31}^{2}=m_{0}^{2}$. Using this dependence we get from (23) and (24) the ratios $f_{e \mu} / f_{e \tau}$ and $f_{\mu \tau} / f_{e \tau}$ as the functions of $m_{0}$ for fixed value of $P\left(\nu_{\mu} \rightarrow \nu_{e}\right)$ (see Fig.2). 
For $P\left(\nu_{\mu} \rightarrow \nu_{e}\right)=1.5 \times 10^{-3}$, (which is in the range of sensitivity of KARMEN and LSND) we find $f_{e \mu} \simeq f_{\mu \tau} \gg f_{e \tau}$ at $m_{0}=5 \mathrm{eV}$. This relation may testify for certain horizontal symmetry. Below $m_{0}=5 \mathrm{eV}$, there is an inverse flavour hierarchy of the couplings, $f_{e \mu} \geq f_{\mu \tau} \gg f_{e \tau}$. For $P\left(\nu_{\mu} \rightarrow \nu_{e}\right) \leq 10^{-4}$ one gets the inverse flavor hierarchy already below $m_{0}=10 \mathrm{eV}$.

Fig. 2

The absolute value of the coupling constants can be fixed by (201). For values of parameters: $\sin \phi \simeq O\left(10^{-1}\right), \tan \beta \simeq O(10), M_{1} \simeq M_{2} \simeq O(500 \mathrm{GeV})$ we get: $f_{e \mu}=10^{-2}-1$. That is the scenario implies quite big couplings constants of the Zee boson.

\section{Constraints from the Electroweak Processes}

Since the constants $f_{e \mu}, f_{\mu \tau}$ are rather big the Zee singlet can give observable contributions to different weak processes. The effective four-fermion Lagrangian induced by the Zee boson exchange can be written (after appropriate Fiertz transformation) as

$$
\frac{G_{F}}{\sqrt{2}} \xi\left[\bar{\nu}_{\mu} \gamma^{\mu}\left(1-\gamma_{5}\right) e \bar{\nu}_{\mu} \gamma_{\mu}\left(1-\gamma_{5}\right) e-\bar{\nu}_{\mu} \gamma^{\mu}\left(1-\gamma_{5}\right) \mu \bar{\nu}_{e} \gamma^{\mu}\left(1-\gamma_{5}\right) e+\ldots\right],
$$

where

$$
\xi \equiv\left(\frac{1}{\sqrt{2} G_{F}} \frac{f_{e \mu}^{2}}{\bar{M}^{2}}\right)
$$

and

$$
\frac{1}{\bar{M}_{H}^{2}} \equiv \frac{\cos ^{2} \phi}{M_{1}^{2}}+\frac{\sin ^{2} \phi}{M_{2}^{2}}
$$


Notice that only usual left handed components of leptons participate in the interactions with Zee boson, and therefore the Lagrangian (25) has usual $V-A$ form.

In the case neutrino electron scatterings, $\bar{\nu}_{\mu} e^{-} \rightarrow \bar{\nu}_{\mu} e^{-}$and $\nu_{\mu} e^{-} \rightarrow \nu_{\mu} e^{-}$, the contribution from (25) leads to a change of the $g_{L}^{e}$ coupling: $g_{L}^{e} \rightarrow g_{L}^{e}+\xi$. CHARM II experimental data on $g_{L}$ and $g_{R}$ [15 agree well with predictions of the Standard Model. Therefore $\xi$ should be smaller than the experimental error $\Delta g_{L}^{e}: \xi<\Delta g_{L}^{e}$. Using (26) we have explicitly

$$
\frac{f_{e \mu}^{2}}{\bar{M}^{2}}<0.036 G_{F} .
$$

The Zee singlet exchange leads also to the lepton number violating process $\nu_{\mu} e^{-} \rightarrow$ $\nu_{\tau} e^{-}$which contributes to $\nu_{\mu} e$ scattering incoherently. Its amplitude is proportional to $f_{e \mu} f_{e \tau}$.

The second term in the Lagrangian (25) gives the renormalization of the four fermion coupling $G_{F}$ of the muon decay. Assuming that the effect of the Zee boson on the decay rate is smaller than $0.1 \%$, (so that it does not destroy the agreement in the electroweak precision tests) we find

$$
\frac{f_{e \mu}^{2}}{\bar{M}^{2}}<7 \cdot 10^{-4} G_{F} .
$$

Also the modes of the muon decay with lepton number violation appear: $\mu \rightarrow \nu_{\tau} e \bar{\nu}_{e}$, $\mu \rightarrow \nu_{\mu} e \bar{\nu}_{\tau}, \mu \rightarrow \nu_{\tau} e \bar{\nu}_{\mu}$ which contribute to the total decay rate incoherently.

The result (29) allows one to get the bounds on masses and mixing of scalar bosons. Indeed, using expression for the mass (20) we can find $f_{e \mu}$ as the function of $\phi, \beta$ and $M_{i}$. Substituting $f_{e \mu}=f_{e \mu}\left(\phi, \beta, M_{i}\right)$ into (29) we find the lower bound on $\sin \phi$ as the function of $M_{1}$ for different values of $P\left(\nu_{\mu} \rightarrow \nu_{e}\right), m_{0}, \tan \beta$ and fixed $M_{2}=300 \mathrm{GeV}$ (see Fig.3 (a)-(c)). Notice that the most strong bound is for $M_{1}=M_{2}$. Forbidden region becomes larger with increase of $\tan \beta$ as well as with decrease of $m_{0}$ and $P\left(\nu_{\mu} \rightarrow \nu_{e}\right)$. Big region of parameters exists in which all the 
restrictions are satisfied. Furthermore, one of the charged Higgses can be at the level of lower kinematical bound.

\section{Fig. 3 (a), 3 (b), 3 (c)}

The bound on the model follows also from $e-\mu-\tau$ universality. The expected deviation from universality due to the Zee boson contribution is $\left|1-g_{\tau} / g_{\mu}\right| \sim$ $\left(f_{e \mu}^{2}\right) /\left(G_{F} \bar{M}^{2}\right)$, where $g_{\mu}$ and $g_{\tau}$ are the weak coupling constants of the charged currents with $\mu$ and $\tau$. Recent measurement of the branching ratio of the decay $\tau \rightarrow e \bar{\nu}_{e} \nu_{\tau}$ at OPAL [16] gives the ratio of couplings $g_{\tau} / g_{\mu}=1.0025 \pm 0.0060$, and the corresponding bound on the parameters of model is weaker than (29).

The model leads to the radiative decays of the muon $\mu \rightarrow e \gamma$ and neutrino $\nu_{3(2)} \rightarrow \nu_{1} \gamma$ through the one-loop diagram with Zee singlet.

The branching ratio of the $\mu \rightarrow e \gamma$ [1] [14] is

$$
B(\mu \rightarrow e \gamma)=\left(\frac{\alpha}{48 \pi}\right)\left(\frac{f_{e \tau} f_{\mu \tau}}{\bar{M}_{H}^{2} G_{F}}\right)^{2} .
$$

Using (19), (20) and (27) we can express it as $B(\mu \rightarrow e \gamma)=A\left(\sin \phi, M_{i}, \tan \beta\right) m_{0}^{4}$. The branching ratio becomes smaller with increase of $\sin \phi$ and decrease of $\tan \beta$ (see fig. 4). The present experimental upper bound $B<4.9 \times 10^{-11}$ [17 (shown by the horizontal dashed line) will be strengthen soon up to $5 \times 10^{-13}$ by the experiment at MEGA in LAMPF(Los Alamos). Future experiment 18 will push the limit to $3 \times 10^{-14}$. The results from these experiments combined with bounds from precision tests (fig.3) will cover essential part of the parameter space of the model.

\section{Fig. 4}


The life time of $\nu_{i} \rightarrow \nu_{1} \gamma(i=2,3)$ equals [1] [14]:

$$
\tau\left(\nu_{i} \rightarrow \nu_{1}\right)=\left\{\alpha m_{i}^{5}\left[2 \frac{m_{\mu}^{2}}{m_{\tau}^{2}} C_{\mu}\left(1-\frac{C_{\tau}}{C_{\mu}} \cos 2 \theta\right)\right]^{2}\left(1-\frac{m_{1}}{m_{i}}\right)^{3}\right\}^{-1}
$$

where

$$
C_{\ell}=\frac{1}{\ln \left(\frac{M_{H 2}^{2}}{M_{H 1}^{2}}\right)}\left[\frac{1}{M_{H 2}^{2}}\left(\ln \left(\frac{M_{H 2}^{2}}{m_{\ell}^{2}}\right)-1\right)-(2 \rightarrow 1)\right], \quad \ell=\mu, \tau .
$$

The life time $\tau\left(\nu_{i} \rightarrow \nu_{1}\right)$ depends mainly on the charged Higgs scalar masses $M_{1}$ and $M_{2} ; f_{\ell \ell^{\prime}}$ and $\sin \phi$ enter only via the mass of neutrino. For $m_{0}=1-10 \mathrm{eV}$ the life time is in the interval $10^{22}-10^{29}$ years. This may have some cosmological implications.

In the limit of $f_{e \tau}=0$ the anomalous magnetic moment of neutrino which corresponds to (31) equals 14

$$
\mu_{\nu} \simeq-4 e m_{0} C_{\tau}
$$

where $C_{\tau}$ is defined in (32). For $M_{1} \simeq M_{2} \simeq 300 \mathrm{GeV}$ and $m_{0}=2.65 \mathrm{eV}$, we get $\mu_{\nu} \simeq 6 \times 10^{-16} e / 2 m_{e}$

\section{Solar neutrinos}

For solar neutrinos all oscillations are averaged and from (12) one gets survival probability

$$
P\left(\nu_{e} \rightarrow \nu_{e}\right)=\cos ^{4} \theta+\frac{1}{2} \sin ^{4} \theta+O\left(\epsilon^{2}\right)
$$

There is no dependence of suppression of the $\nu_{e}$ - flux on energy and for $\epsilon, \sin ^{2} \theta \ll 1$ the effect is small. Thus in the considered scenario there is no solution of the solar neutrino problem.

Let us suggest that apart from three known neutrinos also singlet (because of the LEP bound) neutrino $\nu_{s}$ exists. This neutrino mixes with electron neutrino so 
that the resonance conversion $\nu_{e} \rightarrow \nu_{s}$ explains the deficit of the solar $\nu_{e}$-flux. The explanation requires the mass squared difference and the mixing angle in the intervals [19] 20]:

$$
\Delta m^{2}=(4-10) \cdot 10^{-6} \mathrm{eV}^{2}, \quad \sin ^{2} 2 \theta_{e s}=10^{-3}-10^{-2} .
$$

The singlet neutrino could be the right handed counterpart of the known neutrino components or new very light fermion which comes from some other sector of theory.

The mass of the lightest neutrino in the Zee model (which is essentially the $\nu_{e}$ ) is

$$
m_{1}=m_{0} \epsilon \sin 2 \theta \approx \frac{\Delta m_{a t m}^{2}}{2 m_{H D M}} \sim(1-5) \cdot 10^{-3} \mathrm{eV}
$$

Squared mass $m_{1}^{2}$ is close to $\Delta m^{2}$ desired for solar neutrinos (35). This means that the mass of singlet neutrino, $m_{s}$, should be rather close to $m_{1}$ (recall that for the resonance conversion one needs $\left.m_{s}>m_{1}\right)$ :

$$
\frac{m_{s}-m_{1}}{m_{1}} \approx \frac{\Delta m^{2}}{2 m_{1}^{2}} .
$$

For $m_{1}>4 \cdot 10^{-3} \mathrm{eV}$ one gets from this equation $\Delta m / m_{1}<0.2$.

Let us consider the simplest scheme with only one singlet neutrino. We extend the Lagrangian of the Zee model by adding the terms:

$$
f_{l} \bar{\Psi}_{l} \Phi \nu_{s}+m_{s s} \nu_{s}^{T} \nu_{s}
$$

All couplings $f_{i}$ can be of the same order. The first term leads to mixing of the $\nu_{s}$ with the active neutrinos: $m_{l s}=f_{l}\langle\Phi\rangle$. Performing block diagonalization of $4 \times 4$ mass matrix we get the mass matrix for the $\left(\nu_{s}-\nu_{e}\right)$ system:

$$
M \approx\left(\begin{array}{ll}
m_{s s} & m_{e s} \\
m_{e s} & m_{1}
\end{array}\right),
$$

where $m_{e s}=f_{e}\langle\Phi\rangle$ and $m_{1}$ is fixed in (36). The mixing angle is then

$$
\sin 2 \theta_{e s} \approx \frac{2 m_{e s}}{m_{s s}-m_{1}}
$$


If the mass $m_{s s}$ is not too close to $m_{1}$, we get

$$
m_{s} \sim(2-4) \cdot 10^{-3} \mathrm{eV}, \quad m_{e s} \sim 10^{-4} \mathrm{eV} .
$$

With increase of $m_{s}$ (and consequently the degeneracy) $m_{e s}$ can be further diminished.

According to (41) a solution of the solar neutrino problem implies very small

Yukawa coupling $f_{e}<10^{-15}$ which is of the order $m_{E W} / m_{\text {string }}$, where $m_{\text {string }} \sim 10^{18}$ $\mathrm{GeV}$ is the superstring scale. The mass of the singlet neutrino $m_{s}$ is of the order $m_{3 / 2}^{2} / m_{\text {string }}$ which also may indicate the SUSY origin of the singlet.

Mixing of the singlet neutrino with high mass states $\nu_{\mu}, \nu_{\tau}$ is of the order

$$
\sin ^{2} 2 \theta_{\mu s} \sim \sin ^{2} 2 \theta_{e s} \cdot \sin ^{2} \theta \sim 10^{-7},
$$

so that the bound from the primordial nucleosynthesis [21] can be satisfied.

The influence of the singlet fermion on "standard" structure of the Zee model is negligibly small and the results of the previous sections are not changed.

\section{Conclusions}

1. Zee model reproduces rather naturally the pattern of neutrino masses and mixing which solves the atmospheric neutrino problem, supplies a desired HDM component in the Universe and gives the $\bar{\nu}_{\mu}-\bar{\nu}_{e}$ oscillations in the range of sensitivity of existing experiments.

2. The solar neutrino problem can be solved in extension of the model with an additional singlet fermion $s$, so that solar neutrinos undergo $\nu_{e} \rightarrow s$ conversion. The introduction of $s$ does not destroy basic features of the Zee model.

3. The data on oscillations of solar and atmospheric neutrinos as well as the cosmological mass scale fix all parameters of the Zee mass matrix. The scenario 
implies in general inverse flavour hierarchy of the Zee boson couplings. There is a possibility of $f_{e \mu} \simeq f_{\mu \tau} \gg f_{e \tau}$ which may imply certain horizontal symmetry.

4. The masses of the charged scalar bosons are of the order $100-500 \mathrm{GeV}$, and in certain cases at least one of the bosons can be as light as the lower kinematical bound.

5. The scenario will be tested in forthcoming experiments:

(i) the probability of $\nu_{\mu} \rightarrow \nu_{\tau}$ oscillations is expected to be very small; discovery of these oscillations in CHORUS and NOMAD will rule out the scenario. (ii) The signal of $\nu_{e} \rightarrow \nu_{\tau}$ oscillations may be in the region of sensitivity of these experiments. (iii) The confirmation of the LSND positive result will further testify for the suggested scenario. (iv) One may expect deviations from the SM predictions in $\mu \rightarrow \nu_{\mu} e \bar{\nu}_{e}$ decay, $\bar{\nu}_{\mu} e^{-} \rightarrow \bar{\nu}_{\mu} e^{-}$and $\nu_{\mu} e^{-} \rightarrow \nu_{\mu} e^{-}$scatterings, the violation of $e-\mu-\tau$

universality etc.. (v) The $\mu \rightarrow e \gamma$ decay can be close to the present experimental upper bound. (vi) The life time of the neutrino radiative decay $\nu_{3(2)} \rightarrow \nu_{1} \gamma$ is expected to be $10^{22}-10^{29}$ years. The decay of the relic neutrinos may have observable astrophysical consequences.

\section{Acknowledgments}

We would like to thank Z. Hioki, K. Hagiwara and K. Hikasa for the helpful discussion of radiative corrections. We also thanks H. Shibuya for experimental informations of $\nu_{e} \rightarrow \nu_{\tau}$ at CHORUS. This research is supported by the Grant-in-Aid for Science Research, Ministry of Education, Science and Culture, Japan(No. 07640413). 


\section{References}

[1] A. Zee, Phys. Lett. 93B(1980)389; ibidem 161B(1985)141.

[2] M. Gell-Mann, P. Ramond and R. Slansky, in Supergravity, Proceedings of the Workshop, Stony Brook, New York, 1979, edited by P. van Nieuwenhuizen and D. Freedmann, North-Holland, Amsterdam, 1979, p.315;

T. Yanagida, in Proceedings of the Workshop on the Unified Theories and Baryon Number in the Universe, Tsukuba, Japan, 1979, edited by O. Sawada and A. Sugamoto, KEK Report No. 79-18, Tsukuba, 1979, p.95.

[3] A.Y. Smirnov and Z. Tao, Nucl. Phys. B426(1994)415.

[4] K.S. Hirata et al., Phys. Lett. 205B(1988)416; 280B(1992)146;

D. Casper et al., Phys. Rev. Lett. 66(1991)2561;

R. Becker-Szendy et al., Phys. Rev. D46(1992)3720;

NUSEX Collaboration, Europhys. Lett. 8(1989)611; ibidem 15(1991)559;

SOUDAN2 Collaboration, Nucl. Phys. B35(Proc. Suppl.)(1994)427;

ibidem 38(1995)337;

Fréjus Collaboration, Z. Phys. C66(1995)417;

MACRO Collaboration, Phys. Lett. 357B(1995)481;

Y. Fukuda et al., Phys. Lett. 335B(1994)237.

[5] R. N. Mohapatra and G. Senjanovic, Z. Phys. C17(1983) 53;

R. Holman, G. Lazarides and Q. Shafi, Phys. Rev. D27(1983) 995;

Q. Shafi and F. W. Stecker, Phys. Rev. Lett. 53(1984) 1292;

J.R. Primack, J. Holtzman, A. Klypin and D. O. Caldwell, Phys. Rev. Lett. $\mathbf{7 4}(1995) 2160$.

[6] E776 Collaboration, L. Borodovsky et al., Phys. Rev. Lett. 68(1992)274. 
[7] KARMEN Collaboration, Nucl. Phys. B38(Proc. Suppl.)(1995)235.

[8] L. Wolfenstein, preprint at Carnegie Mellon University, CMU-HEP95-05(1995).

[9] A. Joshipura and A. Smirnov, 1994 unpublished.

[10] LSND Collaboration, C. Athanassopoulos et al., Phys. Rev. Lett. 75(1995)2650; J. E. Hill, Phys. Rev. Lett. 75(1995)2654.

[11] K. Winter, Nucl. Phys. B38(Proc. Suppl.)(1995)211;

M. Baldo-Ceolin, ibidem 35(1994)450;

L. DiLella, Nucl. Phys. B31(Proc. Suppl.)(1993)319.

[12] L. Camilleri, Neutrino Oscillation Experiments at Accelerators, CERN-PPE/9487(1994).

[13] G.M. Fuller, R. Mayle, B.S. Meyer and J.R. Wilson, The Astrophysical Journal, 389(1992)517;

Y-Z. Qian, G.M. Fuller, G.J. Mathews, R.W. Mayle, J.R. Wilson and S.E. Woosley; Phys. Rev. Lett. 71(1993)1965.

[14] S.T. Petcov, Phys. Lett. 115B(1982)401;

S.M. Bilenky and S.T. Petcov, Rev. Mod. Phys. 59(1987)671.

[15] CHARM II Collaboration, P. Vilain et al., Phys. Lett. 281B(1992)159.

[16] The OPAL Collaboration, CERN-PPE/95-142(1995).

[17] Particle Data Group, Phys. Rev. D50(1994)1173(1994).

[18] Y. Kuno, Invited talk at KEK meeting "CP violation and its origin" (1996). 
[19] GALLEX Collaboration, Phys. Lett. 327B(1994)377;

SAGE Collaboration, Phys. Lett. 328B(1994)234;

Homestake Collaboration, Nucl. Phys. B38(Proc. Suppl.)(1995)47;

Kamiokande Collaboration, Nucl. Phys. B38(Proc. Suppl.)(1995)55.

[20] S.P. Mikheyev and A.Yu. Smirnov, Yad. Fiz. 42(1985)1441;

L. Wolfenstein, Phys. Rev. D17(1987)2369;

E.W. Kolb, M.S. Turner and T.P. Walker, Phys. Lett. 175B(1986)478;

S.P. Rosen and J.M. Gelb, Phys. Rev. D34(1986)969;

J.N. Bahcall and H.A. Bethe, Phys. Rev. Lett. 65(1990)2233;

N. Hata and P. Langacker, Phys. Rev. D50(1994)632;

P.I. Krastev and A. Yu. Smirnov, Phys. Lett. 338B(1994)282.

[21] For recent discussion see e.g. C. Y. Cardall, G. Fuller, astro-ph/9603105(1996). 


\section{Figure Captions}

Fig.1 The dependence of the oscillation probabilities $P\left(\nu_{e} \rightarrow \nu_{\tau}\right)$ at CHORUS and NOMAD (solid line), and $P\left(\nu_{\mu} \rightarrow \nu_{e}\right)$ at LSND (dashed line) on $\sin \theta$ for $\Delta m_{32}^{2}=10^{-2} \mathrm{eV}^{2}$ and $m_{0}=2.45 \mathrm{eV}$.

Fig.2 The ratios $f_{\mu \tau} / f_{e \tau}$ (solid line) and $f_{e \mu} / f_{e \tau}$ (dashed line) as the functions of $m_{0}$ for $P\left(\nu_{\mu} \rightarrow \nu_{e}\right)=1.5 \times 10^{-3}$.

Fig.3 The lower bound for $\sin \phi$ as the function of $M_{1}$ for $P\left(\nu_{\mu} \rightarrow \nu_{e}\right)=$ $3 \times 10^{-3}$ (solid curve), $3 \times 10^{-4}$ (long-dashed curve) and $3 \times 10^{-5}$ (short-dashed curve). For other parameters we take (a) $\tan \beta=59.3, m_{0}=2.45 \mathrm{eV}$, (b) $\tan \beta=2$, $m_{0}=2.45 \mathrm{eV}$ (c) $\tan \beta=59.3, m_{0}=10 \mathrm{eV}$. In all the cases $M_{2}=300 \mathrm{GeV}$.

Fig.4 The dependence of the branching ratio of $\mu \rightarrow e \gamma$ on $m_{0}$ for $\sin \phi=0.02$ (solid curve), 0.05 (dashed curve), and 0.1(dashed-dotted curve). The values of other parameters are fixed as $\tan \beta=20, M_{1}=500 \mathrm{GeV}, M_{2}=300 \mathrm{GeV}$ and $\sin ^{2} 2 \theta=2 \times 10^{-3}$. The short vertical lines indicate the lower bounds on $m_{0}$ from the muon decay. The experimental upper bound on $B(\mu \rightarrow e \gamma)$ is shown by the horizontal dashed line. 
This figure "fig1-1.png" is available in "png" format from: http://arxiv.org/ps/hep-ph/9604370v1 
This figure "fig1-2.png" is available in "png" format from: http://arxiv.org/ps/hep-ph/9604370v1 
This figure "fig1-3.png" is available in "png" format from: http://arxiv.org/ps/hep-ph/9604370v1 